\title{
ОБ ОДНОЙ ПОСТАНОВКЕ ЗАДАЧИ ОПТИМИЗАЦИИ ИЕРАРХИЧЕСКИХ СТРУКТУР
}

\author{
T. RIISMAA. OHEST HIERARHILISTE STRUKTUURIDE OPTIMEERIMISE OLESANDE ESITUSEST \\ T. RIISMAA. ON A STATEMENT OF THE OPTIMIZATION PROBLEM FOR HIERARCHICAL \\ STRUCTURES
}

Конечный граф $(E, U)$ есть прадерево с корнем $x_{1} \in E$, если дуга;

1) в каждую его вершину, кроме $x_{1}$, заходит одна единственная

2) в $x_{1}$ не заходит ни одна дуга;

$3)(E, U)$ не содержит контуров $\left(\left[{ }^{1}\right]\right.$, c. 173$)$.

Предположим, что множество вершин $E$ разделено на два непустых непересекающихся множества $E=C \cup P: C=\{x \in E \mid \Gamma x \neq \varnothing\}$ и $P=$ $=\{x \in E \mid \Gamma x=\varnothing\}$, где $\Gamma x=\{y \mid(x, y) \in U\}$ и $x, y \in E,(x, y)$ - дуга графа, $U-$ множество дуг.

Множество $C=\left\{c_{1}, \ldots, c_{m}\right\}$ будем называть множеством узлов (невисящих вершин), $P=\left\{p_{1}, \ldots, p_{n}\right\}-$ множеством точек (висящих вершин) ([ $\left.{ }^{1}\right]$, с. 165$)$.

На всяком подмножестве $F \subset E$ определим всевозможные прадеревья, множеством вершин которых является это подмножество. Получим множество прадеревьев $H_{E}$. Для $F$ предполагается

$$
F=F_{1} \cup F_{2}, F_{1} \subset C, F_{2} \subset P, F_{2} \neq \varnothing .
$$

Таким образом, множество узлов любого прадерева $H \in H_{E}$ является подмножеством множества $C$, а множество точек - подмножеством множества $P$.

Каждой точке $p_{i} \in P$ поставим в соответствие функцию $f_{i}\left(x_{i}\right)$, где $f_{i}: R_{+} \rightarrow R_{+}, f_{i}(0)=0(i=1, \ldots, n)$. Пусть $x_{i}=\left(x_{i 1}, \ldots, x_{i q}\right)$ и $Q=$ $=\left\{x=\left(x_{1}, \ldots, x_{n}\right) \mid G(x) \geqslant 0, x \geqslant 0\right\} . \quad$ Каждому узлу $c_{j} \in C$ поставим в соответствие функцию $h_{j}\left(k_{j}\right)$, где $h_{j}: N \rightarrow R_{+}, h_{j}(0)=0, h_{j}\left(k_{j}\right)>0$ при $k_{j}>0$ и $h_{j}-$ возрастающая функция $(j=1, \ldots, m), N=$ $=\{0,1, \ldots\}$. Величина $k_{j}$ интерпретируется как число дуг, исходящих из узла $c_{j}$.

Далее, прадерево будем называть иерархией. Каждой иерархии $H \in H_{E}$ со множеством узлов и точек $F=F_{1} \cup F_{2}=\left\{c_{\tau_{1}}, \ldots, c_{\tau_{\alpha}}\right\} \bigcup$ $\cup\left\{p_{v_{1}}, \ldots, p_{v_{\beta}}\right\}$ сопоставлена функция

$$
\varphi(H)=f * h=f_{v_{1}} * \ldots * f_{v_{\beta}} * h_{\tau_{1}} * \ldots * h_{\tau_{\alpha}},
$$

где * означает некоторый закон синтеза, $v_{1}, \ldots, v_{\beta}$ и $\tau_{1}, \ldots, \tau_{\alpha}$ явля- 
ются подпоследовательностями последовательностей $1, \ldots, n$ и $1, \ldots$ ..., $m$ соответственно.

В качестве такого закона рассмотрим соотношение

$$
f * h=\sum_{i=1}^{\beta} f_{v_{i}}\left(x_{v_{i}}\right)-\sum_{j=1}^{\alpha} h_{\tau_{j}}\left(k_{\tau_{j}}\right) .
$$

Иерархия $H_{0}$, при которой

$$
\varphi\left(H_{0}\right)=\max _{H \in H_{E}} \varphi(H),
$$

называется оптимальной иерархией.

Иерархия интерпретируется как структура иерархической системы, которая состоит из координаторов (узлов), процессов (точек) и соотношений между ними (дуг), направление которых показывает определенную подчиненность этих узлов и точек.

Первое слагаемое в соотношении (1) интерпретируется как продукция $\beta$ процессов $(\beta \leqslant n)$, а второе - как затраты на координирование работы $\beta$ процессов и $\alpha-1$ координаторов $(\alpha \leqslant m)$. Величина $x_{i}$ может быть рассмотрена как ресурсы в распоряжении процесса $p_{i}$, а величина $k_{j}$ - как координаторы и процессы, подчиненные координатору $c_{j}$.

Задача нахождения оптимальной иерархии сводится к решению следующей задачи частично целочисленного нелинейного программирования: по $x_{1}, \ldots, x_{n}, \beta, k_{1}, \ldots, k_{m}, \alpha$ максимизировать

$$
\sum_{i=1}^{n} f_{i}\left(x_{i}\right)-\sum_{j=1}^{m} h_{j}\left(k_{j}\right)
$$

при условиях

$$
\begin{aligned}
& \sum_{j=1}^{m} k_{j}=\beta+\alpha-1, \\
& \sum_{j=1}^{m} \delta\left(k_{j}\right)=\alpha, \quad \sum_{i=1}^{n} \delta\left(x_{i}\right)=\beta, \\
& \alpha \in N, \quad \beta \in N \backslash\{0\}, \quad k_{j} \in N \quad(j=1, \ldots, m), \\
& x \in Q,
\end{aligned}
$$

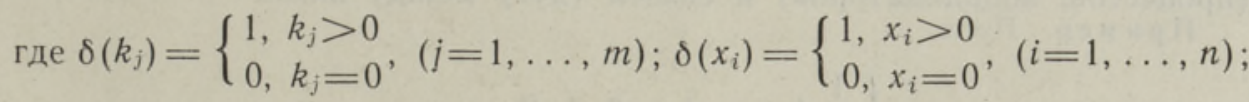
$x=\left(x_{1}, \ldots, x_{n}\right)$.

Здесь $\alpha-$ число узлов $(\alpha \leqslant m)$, а $\beta-$ число точек $(\beta \leqslant n)$ иерархии. Левая часть ограничения (4) представляет собой те узлы, откуда дуги исходят, а правая - те узлы и точки, куда они заходят. В один узел это корень иерархии - не заходит ни одна дуга. Ограничения (5) показывают, что у иерархии есть $\alpha$ узлов и $\beta$ точек. Из определения $\delta\left(x_{i}\right)$ ясно, что иерархия имеет точку $p_{i}$ тогда и только тогда, когда $x_{i}>0$. Из определения $\delta\left(k_{j}\right)$ ясно, что иерархия имеет узел $\epsilon_{\vec{j}}$ тогда и только тогда, когда $k_{j}>0$. Естественно, что $\alpha, \beta, k_{j}(j=1, \ldots, m)$ неотрицательные целые числа, и из предположения $F_{2} \neq \varnothing$ следует: $\beta \geqslant 1$.

Решение задачи (3)-(7) обозначим через $\left(k^{0}, x^{0}\right)$, где $k^{0}=$ $=\left(k_{1}{ }^{0}, \ldots, k_{m}{ }^{0}, \alpha^{0}\right), x^{0}=\left(x_{1}^{0}, \ldots, x_{n}{ }^{0}, \beta^{0}\right)$. 
Каждой иерархии $H$ из множества $H_{E}$ соответствует допустимое решение задачи (3)-(7) и, наоборот, каждому допустимому решению задачи $(3)-(7)$ соответствует некоторая иерархия из множества $H_{E}$.

Допустимое решение $(k, x)$ задачи $(3)-(7)$ не определяет иерархию полностью в том смысле, что каждому допустимому решению может соответствовать целое множество иерархий. Вектор $k=$ $=\left(k_{1}, \ldots, k_{m}, \alpha\right)$ определяет множество узлов иерархии и число дуг, исходящих из каждого узла. Вектор $x=\left(x_{1}, \ldots, x_{n}, \beta\right)$ определяет множество точек иерархии. Всевозможные иерархии, имеющие вышеуказанное множество точек, узлов и число дуг, исходящих из каждого узла и заданных положительными компонентами вектора $k$, соответствуют допустимому решению $(k, x)$ задачи $(3)-(7)$.

Иногда возникает задача о нахождении оптимальной иерархии, где число точек $\beta$ фиксировано заранее. Тогда задача (3)-(7) распадается на две самостоятельные задачи:

$$
\max _{x_{i}, \ldots, x_{n}}\left\{\sum_{i=1}^{n} f_{i}\left(x_{i}\right) \mid x \in Q, \sum_{i=1}^{n} \delta\left(x_{i}\right)=\beta\right\}
$$

и

$$
\begin{aligned}
& \min _{\substack{h_{1}, \ldots, k_{m}, \alpha\\
}}\left\{\sum_{j=1}^{m} h_{j}\left(k_{j}\right) \mid \sum_{j=1}^{m} k_{j}=\beta+\alpha-1,\right. \\
& \left.\sum_{j=1}^{m} \delta\left(k_{j}\right)=\alpha, \alpha \in N, k_{j} \in N \quad(j=1, \ldots, m)\right\},
\end{aligned}
$$

где $\delta\left(x_{i}\right)(i=1, \ldots, n)$ и $\delta\left(k_{j}\right)(j=1, \ldots, m)$ уже определенные функцин.

Задача (8) является задачей нелинейного программирования с дополнительным условием, что положительными могут быть точно $\beta$ компонент. Задача целочисленного нелинейного программирования (9) является задачей нахождения оптимальной иерархии при фиксированном числе точек. Для решения задачи (9) можно использовать метод динамического программирования $\left(\left[^{2}\right]\right.$, с. 251$)$.

Развитый здесь подход к задачам оптимизации иерархии представляет собой одно из возможных продолжений идеи Р. Куликовского $\left[{ }^{3}\right]$, который рассматривал иерархии, состоящие из объектов двух типов (процессов, координаторов) и связей (дуг) между ними.

При мер. Пусть

$$
\begin{gathered}
h_{1}\left(k_{1}\right)= \begin{cases}\frac{1}{2} k_{1}, \quad k_{1}=0,1,2, & h_{2}\left(k_{2}\right)=k_{2}^{3 / 2}, \\
8 k_{1}-15, & k_{1} \geqslant 3,\end{cases} \\
h_{3}\left(k_{3}\right)=k_{3}^{2}, \quad h_{4}\left(k_{4}\right)=4 k_{4},
\end{gathered}
$$

где $k_{j} \in N(j=1, \ldots, 4)$, и $m=4$. Зафиксировано: $\beta=6$.

При этих данных задача (9) имеет вид

$$
\begin{aligned}
& \min _{k_{1}, \ldots, k_{*}, \alpha}\left\{\sum_{j=1}^{4} h_{j}\left(k_{j}\right) \mid \sum_{j=1}^{4} k_{j}=5+\alpha,\right. \\
& \left.\qquad \sum_{j=1}^{4} \delta\left(k_{j}\right)=\alpha, \alpha \in N, k_{j} \in N(j=1, \ldots, 4)\right\},
\end{aligned}
$$

ее решешисм является вектор $k^{0}=\left(k_{1}{ }^{0}, \ldots, k_{4}{ }^{0}, \alpha^{0}\right)=(2,3,3,0,3)$. 


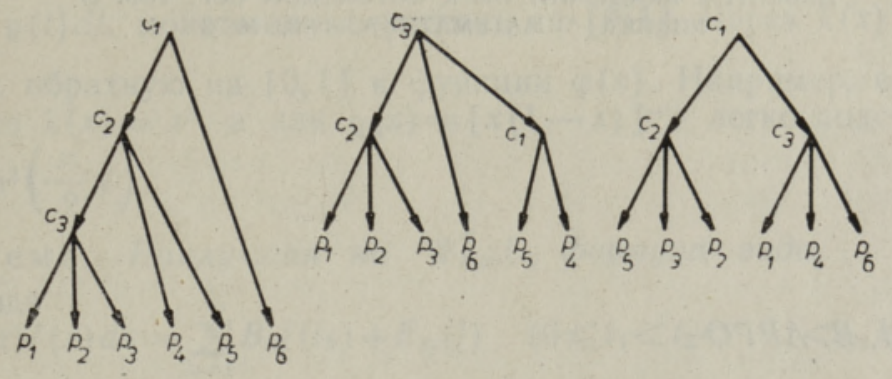

На рисунке показаны некоторые иерархии, соответствующие решению $k^{0}$.

\section{Л И Т Е Р А Т Р Р А}

1. Бер ж К., Теория графов и ее применения, М., 1962.

2. Корбут А. А., Финкельш тейн Ю. Ю., Дискретное программирование, М., 1969.

3. Kulikowski, R., Arch. Automat. i Telemech., 12, № 3, 295 (1967).

Ннститут кибернетики

Академии наук Эстонской ССР
Поступила в редакцию 1/VII 1977 